\title{
Route maps for implant positioning: a review
}

\author{
Manaswini Draksharapu 1,*, Chalapathi Rao Duggineni², Ravi Kumar C2, Harilal G3 \\ ${ }^{1}$ Postgraduate Student, ${ }^{2}$ Professor, ${ }^{3}$ Reader, Mamata Dental College, Giri Prasaad Nagar, Khammam, \\ Telangana, India -507002 .
}

\section{N F O R M A T I O N A B S T R A C T}

\section{Article History}

Received 15 ${ }^{\text {th }}$ March 2021

Received revised 3rd May2021

Accepted 10th May 2021

Available online

15 $5^{\text {th }}$ May 2021

\section{K E Y W O R D S}

Implant placement

Surgical guide

Surgical template,

Cone beam computed tomography

Stereolithography
Dental implants are becoming a more common treatment choice in recent years, with an increasing number of patients preferring this option. Proper implant positioning is the most significant requirement for a successful implant treatment prognosis. A transfer system is necessary to ensure logical continuity between the diagnosis, prosthetic preparation, and surgical phases. Various techniques have been proposed for the fabrication of surgical guide templates in implant dentistry. This paper aims to review the associated literature and recent advancements in this field based on the design concept.

\section{Introduction}

The precise placement of the dental implant is essential to accomplish a pleasing result and the proper alignment to withstand occlusal forces. Dental implants inserted with a surgical guide are more precisely positioned than those placed without a guide. An implant placement guide also makes surgery less stressful for the surgeon because the critical placement factors were taken into account during the fabrication of the surgical guide. A precise reference for implant placement is provided by a stable and accurate surgical guide [1-3].

The use of prosthodontic terms and nomenclature in defining radiographic and surgical models are currently very confusing. The terms stent, guides, model, and equipment were used in the definition of these prostheses. Other terminologies often used in the identification of these prostheses include scanning equipment and radiographic equipment. [4]

\section{Surgical template classification}

Surgical and radiographic templates may be categorised by the type of material used in the prosthesis fabrication and the amount of restriction (drill guidance) associated with the template [5,6].

\subsection{Materials}

\subsubsection{Clear vacuum-formed matrices}

There are numerous advantages of clear vacuum-formed matrices, including cost efficiency, manufacturing ease, translucency, and variability. In addition to the clear matrix, many different materials were used, such as auto polymerizing acrylic resin, guttapercha, and metal rods.

\subsubsection{Autopolymerized acrylic resin}

These are composed with Poly(methyl methcrylate) resins with modifiers. 
2.1.3 Metallic guides of light polymerising composite resins

Metallic guides of light polymerising composite resins closely fit the diameter of drills or implant size. These guides are fabricated with the aid of computer-aided design/computer-aided manufacturing (CAD/CAM) technology. The accuracy and ease of manufacturing have been expanded using interactive treatment planning via CBCT imaging [7-9].

Three different surgical guide designs depending on supporting surfaces have been described [10]. They include i. tooth-supported surgical guide is placed on the remaining natural teeth, ii. mucosa-supported surgical guide is directly placed on the mucosa, allowing flapless implant placement, and iii. bonesupported surgical guide is placed on the bone following a full-thickness mucoperiosteal flap elevation.

\subsection{The principles to design the Surgical guide templates $[11,12]$}

The principles to design the Surgical guide templates are nonlimiting design, partially limiting design, and completely limiting design. These design principles are categorised based on the amount of surgical restriction offered by surgical guide templates.

\subsection{Nonlimiting design}

Nonlimiting designs only indicate to the surgeon where the proposed prosthesis is in relation to the selected implant site. This design suggests the ideal location of the implants without any emphasis on the angulation of the drill. Thus, allowing too much flexibility in the final positioning of the implant.

A summary of Blustein et al. (1986) [13] and Engelman et al. (1988) [14] was the technique of perforating a guide pinhole through a transparent vacuum-formed matrix. This guide pin hole suggested the optimum location of the dental implant. The angulation, however, was measured by the use of adjacent and opposing teeth. The circumference lead strip guide in which a lead strip is attached to the external surfaces of diagnostic waxing was described by Almog et al. (2001) [15].Thiswas used to outline the tooth position over the implant site. It has been observed that the use of these guides can lead to inappropriate access hole placement and/or inappropriate angulation of the implant. Therefore, during the surgical stage of implant placement, these will serve as imaging indicators.

\subsection{Partially limiting design}

The first drill used for osteotomy is guided using the surgical guide in such designs, and the remainder of the osteotomy and implant placement is then performed free-hand by the surgeon. Techniques based on this principle of design include the fabrication of a radiographic template, which is then transformed following radiographic evaluation into a surgical guide template. In the following stages of manufacturing, various authors have proposed various techniques involving modifications, including the material used to manufacture the surgical template, radiographic markers used, the type of imaging device used, and the conversion process involved in transforming the radiographic template into a surgical template.

\subsection{Completely limiting design}

The location, angulation and depth of the osteotomy are determined by guided tubes or sleeves with the complete limiting design, thereby restricting any variation by the implant surgeon. This type of guide can prevent any osteotomy error in the bucco-lingual and mesiodistal plane.

Additionally, drill stops can be incorporated to prevent over-preparing the site. Basically, with the complete limiting design, the final position of the implant is known before the actual surgery. This technique is becoming popular because the final prosthetic abutment or provisional restoration can be prefabricated for immediate provisionalisation after implant placement.

\subsection{Free-hand technique}

With the free-hand technique, the positioning accuracy of guide holes or sleeves is operator based. This approach does not exactly parallel implant positions and has the highest margin of error [16-18].

\subsection{Milling}

Milling is an accurate method in which the guide holes or sleeves are placed using a milling machine. This technique involves special equipment that is primarily used in dental laboratory environments, and precision depends heavily on the expertise of the technician[16].

\subsection{Computer-Aided Design/Computer-Aided manufacturing}

This technique uses three-dimensional images with specialized software to allow the implant guide holes and sleeves to be precisely placed. Using the reformatted CT images of the osseous morphology, done density, 
opposing occlusion, and ideal implant positioning, precise final positioning can be obtained [19]. In a systematic review, John Wiley concluded that various computer-guided template-based implant treatments are available. Different types of software, template production and template stabilization, and variations of the surgical and prosthetic protocol are reported. The survival rate of implants placed with computerguided technology is comparable to conventionally placed implants ranging from $91 \%$ to $100 \%$ after an observation time of 12-60 months [20].

\section{Surgical templates}

The implant body positioning is dictated by the surgical design, which provides the best combination of support for repeated occlusion forces, esthetics, and hygiene requirements.

\subsection{Requirements of a surgical template}

- Should be rigid and stable in the accurate position.

- Should fit over and/or around remaining teeth in the arch to stabilise it in the proper position.

- The template should be able to extend onto un-reflected soft tissue regions if the arch has no remaining teeth. The template can then be used after the implanted soft tissues have been reflected.

- On the diagnostic wax-up, the optimal angulation for implant placement should be determined, and the template should correspond to this location during surgery. For each implant, at least two reference points are needed. The surgical guide must be raised above the edentulous bone.

- The distance between two points on the proposed abutment crown's occlusal surface (central fossa or incisal edge) and the crest of the ridge is approximately $8 \mathrm{~mm}$. Consequently, a line representing the optimal implant insertion path can be drawn between these two points of reference. The perfect angulations are parallel to the most anterior abutment joined to the implant and perpendicular to the occlusal plane.

- Other criteria for fabrication of the surgical template include size, transparency, surgical asepsis, and the ability to revise the template as indicated.

- The template should not be too bulky and difficult to insert, or it should not obscure nearby landmarks.

- During bone grafts or implant placement, the surgical design must not contaminate the surgical area. It should be transparent so that when the template is in position, the bony ridge and drills can be more readily seen.

- The surgical outline must correspond to the desired facial contour. Since many edentulous ridges have lost facial bone, the template may help to decide how much augmentation is required for implant placement or lip and face support.

- The surgical template can be used in combination with a bone graft, and then the same template can be used for implant insertion and positioning.

Ideally, the surgical guide should possess the following characteristics:

- Simple and cost-effective to fabricate.

- Stable retention in surgical field (adjacent teeth or landmark).

- Easy access of drills/guide pins/osteotomes intraoperatively.

- Ability to translate pre-surgical work-up information accurately to the operating field.

The manufacture of the surgical guide template involves an arrangement of the diagnostic tooth by one of the following methods.

i. Diagnostic wax-up

ii. Checkingthe arrangement of denture teeth, or

iii. The duplication of the patient's pre-existing dentition/restoration.

\section{Rationale for radiographic template}

The optimal location of the final tooth position or prosthesis must be determined in order to correlate the positioning of the implant with the available bone. The implant may be surgically positioned in an inappropriate position without a specific location, resulting in biomechanical problems with potential complications. A correlation between the tooth or prosthesis location and the radiographic survey must exist to obtain this information. The ideal implant positioning will be complex and maybe difficult if no correlation exists [21].

\subsection{Fabrication of radiographic template (Scanning template)}

4.1.1Radiopaque Markers

A radiopaque material must be used to correlate tooth location and tissue in relation to available bone and vital structures. Barium sulphate $\left(\mathrm{BaSO}_{4}\right)$, an inorganic 


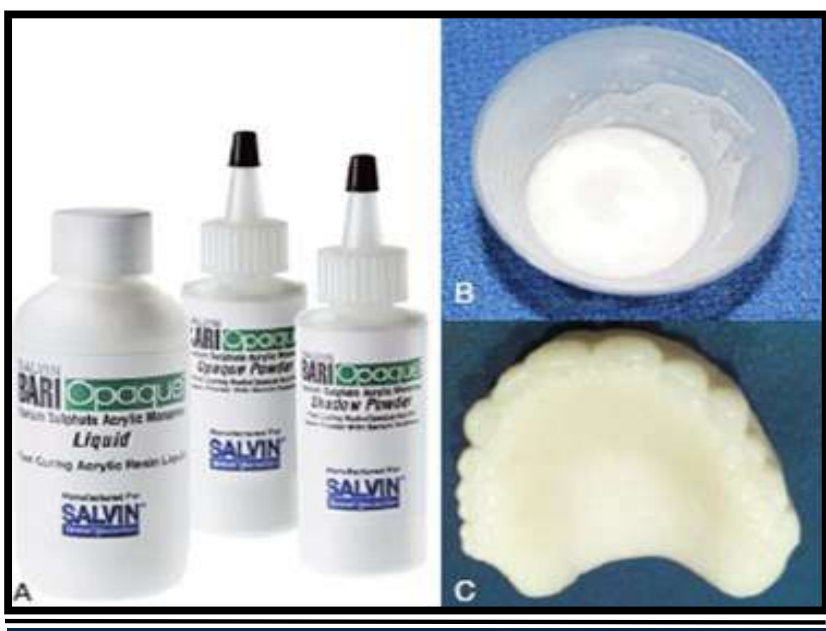

Figure 1. A. Barium sulphate, B. Ideal

homogenous mixture, C. Barium sulphate duplicated prosthesis

compound used clinically as a radio-contrast material in medical diagnostic imaging, is the most common material used today in implant dentistry. Techniques to incorporate $\mathrm{BaSO} 4$ into the radiographic template include (Figure 1) filling the edentulous area with $\mathrm{BaSO}_{4}$, painting the outside aspects of the buccal and lingual surfaces of the template, and use of preformed $\mathrm{BaSO}_{4}$ teeth. Care must be made not to use too high of a concentration of $\mathrm{BaSO}_{4}$ because it may cause excessive scatter in the scan [22,23].

\subsection{Single- scan versus Double-scan technique}

A radiopaque prototype with a single-scan technique (discussed later for partial and completely edentulous) is used by most planning software today. The composition of the radiopaque prototype and its functionality depending on the type of software used during the planning process. The protocol should be obtained before the scan to avoid problems with incorporating the CT data into the scanning software.

\subsubsection{Single scan}

Barium sulphate is used in a 20 per cent $\mathrm{BaSO}_{4}$ solution to recognise the teeth from the diagnostic wax -up. If a soft tissue (flapless surgery) template is to be made, teeth are ideally identified with a $20 \%$ BaSO4 solution, and the base (soft tissue) uses a $10 \%$ mix. This helps teeth to be separated from soft tissue. A non -homogeneous mixture that exhibits areas of high radiolucency can result in poor mixing [24].

\subsubsection{Double scan}

It exhibits less scatter than the single scan technique. In this technique, reference markers (radiopaque

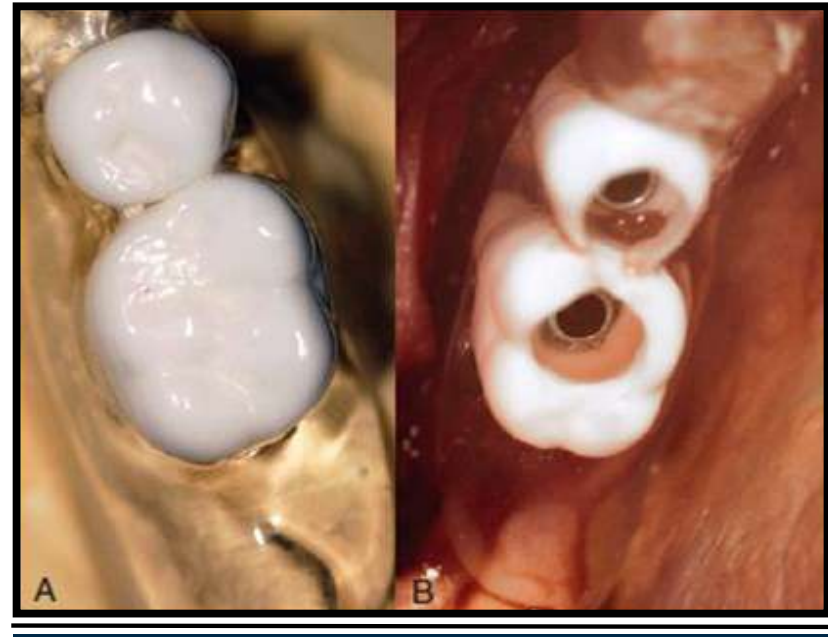

Figure 2. Radiographic template (RT).

Where, A. RT is transformed into a surgical Template, and B. after completion of the scan.

material) are embedded into the radiopaque template. The patient is scanned wearing the template, and then the template is removed from the patient's mouth and scanned separately. The software program uses the markers to correlate the images. With the double-scan technique, the denture base resin and artificial teeth can be reconstructed for planning purposes. The soft tissue can be determined as the difference between the template and bone. The number and location of markers depend on the software program being used [25].

\subsection{Fabrication of partially edentulous radiographic template}

It is the most simple technique. The duplicate study cast is made after the fabrication of a diagnostic waxup. A clear vacuum-formed matrix is made. With the use of $\mathrm{BaSO}_{4}$, the material is added to the edentulous site. The patient then wears the prosthesis during the scanning process. A laboratory or an in-office technique may fabricate this prosthesis.

\subsection{Fabrication of Fully edentulous radiographic template}

If the present prosthesis of the patient requires no alteration due to aesthetics or function, the prosthesis is duplicated via a denture duplicator. During the scanning procedure, the patient wears the completely edentulous radiopaque template. Care must be taken so that the prosthesis during the scanning process is stable. Application of denture adhesive is strongly recommended before the scan to prevent inaccuracies in the position of the teeth. 


\subsection{Transforming a Radiographic Template into a} Surgical guide

It is easy to construct a surgical guide from a radiographic template. If the diagnostic wax-up has determined the optimal positioning of the teeth, openings may be made to allow for accurate implant guidance (Figure2). There are two types of techniques for creating surgical guides from the programme for treatment planning. They are photopolymerisation by laser of liquid resin, and around CAD/CAM.

\subsection{Additional forms (guides) of models}

Two different models have been described and they are discussed in the following sections.

\subsubsection{Models with stereolithography}

A laser-dependent rapid polymerisation process using sequential layers of special polymers that can replicate the exact shape of osseous anatomy is the development of stereolithographic models [23]. These types of models are composed of i. versions of the surgical guide used in manufacturing, ii. pre-surgical models used in preoperative evaluation for implant placement, bone grafting, and orthognathic surgery, and iii. bone reduction guides (BRG). BRGs are similar to reduction copings in conventional crown and bridge, helps in reducing osseous height before implant placement.

\subsubsection{Provisional restorations: "immediate smiles"}

Taking the technology developed by CT to the next level involves manufacturing provisional restorations before implant insertion. Second, the implant dentist will create the virtual treatment plan, followed by the manufacturer creating the computer-generated stereolithographic surgical guides. The surgical guide and articulated diagnostic casts are used by a dental laboratory to produce provisional and (in some cases, final) prostheses. The implant dentist then places the implants and abutments using the surgical guide. Then, immediately after the positioning, the provisional (or final) prosthesis is inserted.

\section{Conclusion}

For the long-term success of implant therapy, the diagnostic phase of implant dentistry is very significant. In the fabrication of radiographic and surgical templates, proper mounting and evaluation of study casts are crucial. Evaluation of the edentulous sites and maxilla-mandibular relationships are an invaluable diagnostic tool in determining ideal implant positioning. Implants should ideally be placed with strict guidelines in relation to adjacent teeth, implants, and vital structures and various planes with respect to the edentulous site. With the use of radiographic and surgical templates, precision has been improved, and uncertainty and surgical time have been reduced, thus addressing complex rehabilitation with greater confidence. In addition, predictable positioning allows for better prosthetic outcome by simplifying abutment selection and avoiding complex laboratory fabrication when misalignment must be corrected. Future technical improvements likely will allow dentists to access these technologies while controlling costs, reducing surgical time, and minimising restorative steps.

Conflicts of interest: Authors declared no conflicts of interest.

\section{Financial support: None}

\section{References}

1. Hinckfuss S, Conrad HJ, Lin L, Lunos S, Seong WJ. Effect of surgical guide design and surgeon's experience on the accuracy of implant placement. J Oral Implantol. 2012;38(4):311-23. https://doi.org/10.1563/AAID-JOI-D-10-00046

2. Pawar A, Mittal S, Singh RP, Bakshi R, Sehgal V. A step toward precision: a review on surgical guide templates for dental implants. Int J Sci Study. 2016;3 (11):262-6.

3. Hinckfuss S, Conrad HJ, Lin L, Lunos S, Seong WJ. Effect of surgical guide design and surgeon's experience on the accuracy of implant placement. J Oral Implantol. 2012;38(4):311-23. https://doi.org/10.1563/AAID-JOI-D-10-00046

4. The glossary of prosthodontic terms, J Prosthet Dent. 2005;94(1):10-92. https://doi.org/10.1016/j.prosdent.2005.03.013

5. $\mathrm{Ku} \mathrm{YC,} \mathrm{Shen} \mathrm{YF.} \mathrm{Fabrication} \mathrm{of} \mathrm{a} \mathrm{radiographic} \mathrm{and}$ surgical stent for implants with a vacuum former. J Prosthet Dent. 2000;83(2):252-3. https://doi.org/10.1016/S0022-3913(00)80019-0

6. Weinberg LA. CT scan as a radiologic data base for optimum implant orientation. J Prosthet Dent. 1993;69(4):381-5. https://doi.org/10.1016/0022-3913 (93)90185-Q

7. Becker CM, Kaiser DA, University of Colorado Health Science Center D. Surgical guide for dental implant placement. J Prosthet Dent. 2000;83(2):24851. https://doi.org/10.1016/S0022-3913(00)80018-9

8. Akça K, İplikçioğlu H, Çehreli MC. A surgical guide 
for accurate mesiodistal paralleling of implants in the posterior edentulous mandible. J Prosthet Dent. 2002;87(2):233-5.

https://doi.org/10.1067/mpr.2002.120900

9. Neidlinger J, Lilien BA, KalantSr DC. Surgical implant stent: a design modification and simplified fabrication technique. J Prosthet Dent. 1993;69(1):70-2. https://doi.org/10.1016/0022-3913(93)90243-H

10. Unsal GS, Turkyilmaz I, Lakhia S. Advantages and limitations of implant surgery with $\mathrm{CAD} / \mathrm{CAM}$ surgical guides: a literature review. J Clinical Exp Dent. 2020;12(4):e409. https://doi.org/10.4317/jced.55871

11. Stumpel III LJ. Cast-based guided implant placement: a novel technique. J Prosthet Dent. 2008;100(1):61-9. https://doi.org/10.1016/S0022-3913(08)60140-7

12. D'Souza KM, Aras MA. Types of implant surgical guides in dentistry: a review. J Oral Implantol. 2012 Oct 20;38(5):643-52. https://doi.org/10.1563/AAIDJOI-D-11-00018

13. Blustein R, Jackson R, Rotskoff K, Coy RE, Godar D. Use of splint material in the placement of implants. Int J Oral Maxillofac Implants. 1986;1:47-49.

14. Engelman MJ, Sorensen JA, Moy P. Optimum placement of osseointegrated implants. J Prosthet Dent. 1988;59(4):467-73. https://doi.org/10.1016/00223913(88)90044-3

15. Almog DM, Torrado E, Meitner SW. Fabrication of imaging and surgical guides for dental implants. J Prosthet Dent. 2001;85: 504-508. https:// doi.org/10.1067/mpr.2001.115388

16. Mason WE, Rugani FC. Prosthetically determined implant placement for the partially edentulous ridge: a reality today. J Mich Dent Assoc. 1999;81(9):28-30.

17. $\mathrm{Ku} \mathrm{YC}$, Shen YF. Fabrication of a radiographic and surgical stent for implants with a vacuum former. J Prosthet Dent. 2000;83(2):252-3. https:// doi.org/10.1016/S0022-3913(00)80019-0

18. Lal K, White GS, Morea DN, Wright RF. Use of stereolithographic templates for surgical and prosthodontic implant planning and placement. Part I. The concept. Journal of Prosthodontics: Implant, Esthetic and Reconstructive Dentistry. 2006;15(1):51-8. https://doi.org/10.1111/j.1532-849X.2006.00069.x

19. Schneider D, Marquardt P, Zwahlen M, Jung RE. A systematic review on the accuracy and the clinical outcome of computer-guided template-based implant dentistry. Clin Oral Implants Res. 2009;20:73-86. https://doi.org/10.1111/j.1600-0501.2009.01788.x

20. Misch CE. Dental implant prosthetics-E-book. Elsevier Health Sciences; 2004 Sep 20.

21. Basten $\mathrm{CH}, \mathrm{Kois} \mathrm{JC}$. The use of barium sulfate for implant templates. J Prosthet Dent. 1996;76(4):451-4. https://doi.org/10.1016/S0022-3913(96)90554-5
22. Israelson H, Plemons JM, Watkins P, Sory C. Barium -coated surgical stents and computer-assisted tomography in the preoperative assessment of dental implant patients. Int J Periodontics Restorative Dent. 1992;12(1): 53-61.

23. Rosenfeld AL, Mandelaris GA, Tardieu PB. Prosthetically directed implant placement using computer software to ensure precise placement and predictable prosthetic outcomes. Part 1: diagnostics, imaging, and collaborative accountability. Int $\mathrm{J}$ Periodontics Restorative Dent 2006;26(3):215-21.

24. Maloney K, Bastidas J, Freeman K, Olson TR, Kraut RA. Cone beam computed tomography and SimPlant materialize dental software versus direct measurement of the width and height of the posterior mandible: an anatomic study. J Oral Maxillofac Surg. 2011;69(7):1923-9.

https://doi.org/10.1016/j.joms.2011.01.003

25. Nikzad S, Azari A. A novel stereolithographic surgical guide template for planning treatment involving a mandibular dental implant. J Oral Maxillofac Surg. 2008;66(7):1446-54.

https://doi.org/10.1016/j.joms.2008.03.004 